\title{
DESIGN AND IMPLEMENTATION OF 2D-DCT BY USING ARAI ALGORITHM FOR IMAGE COMPRESSION
}

\author{
Pratiksha R. Kumbhare*1, U.M. Gokhale ${ }^{2}$ \\ *I Department of Electronics \& Telecommunication Engg, GHRITW, Nagpur, India \\ ${ }^{2}$ Department of Electronics \& Telecommunication Engg, GHRITW, Nagpur, India \\ *1Pratikshakumbhare23@gmail.com; ${ }^{2}$ umgokhale@gmail.com
}

*Corresponding Author: -

Email: Pratikshakumbhare23@gmail.com

\begin{abstract}
: -
Discrete Cosine Transform (DCT) exploits cosine functions; it transforms a signal from spatial representation into frequency domain. It is one of the most widely used techniques for the compression of the image. The main goal of image compression using DCT is the reduction or elimination of redundancy in data representation in order to achieve reduction in storage and communication cost. In this work, we proposed the low complexity architecture for the computation of an algebraic integer (AI) based 8-point DCT. The proposed approach is fast and provides low complexity.
\end{abstract}

Keywords: - Algebraic Integer, Discrete Cosine Transform (DCT), Arai DCT algorithm, Image compression

\section{(c) $(\$)$}




\section{INTRODUCTION}

The DCT is a technique that converts a signal spatial domain to frequency domain.DCT has property of higher energy compaction compared to DFT, DST and DWT. Hence, DCT is generally used for image and video compression. The image is first converted into minimum code units. Then 2-D DCT is applied on each block. To calculate the accurate DCT computation the main obstacle is arising in the implementation of irrational coefficient multiplication.[5] Traditionally, DCT is calculated by rounding off the value to an approximate value. As a consequence, in the computation the errors are systematically introduced, leading to the degradation of the signal to noise ratio. The solution for this issue, algebraic integer (AI) encoding has been employed; the main concept of this encoding is to convert irrational numbers into array of integer numbers which is error free computation. Quantization reduces the number of bits needed to store an integer value by reducing the precision of the integer. The proposed architecture based on the low complexity Arai DCT algorithm for 8-point DCT in image processing application because of relatively low computational complexity.[3] Then, the Huffman coding can further compress the data. All operation performs on the scaled output modes.

\section{LITERATURE SURVEY}

Fausto Galvan and et.al [1], proposed a novel algorithm for the calculation of the first quantization steps from double JPEG compressed Images. Hamdy M. Mousa and et.al [2], proposed image compression technique based on conformal mapping transformation. The proposed compression technique is composed from standard JPEG2000 codec, conformal map and inverse conformal transformation.

Arjuna Madanayake and et.al [3], proposed an algebraic integer (AI)-based two final reconstruction step (FRS) algorithms and time-multiplexed row-parallel architecture for the implementation of bivariate AI- encoded 2-D discrete cosine transform (DCT).

Smitha Joyce Pinto and et.al [4], proposed comparative analysis of compression methods. The comparison of compression methods such as JEPG, JEPG 2000 with SPIHT encoding on the basis of two parameters compression ratio and compression quality.

H. L. P. Arjuna and et.at [5], proposed a time-multiplexed row-parallel architecture for the real-time implementation of bivariate algebraic integer (AI) encoded 2-D discrete cosine transform (DCT) of images.

Hatim Anas and et.al [6], proposed several algorithms for the implementation of the 2D-DCT.The scaled SDCT algorithm is an improve version of the DCT-1D, which consists in gathering all the multiplications at the end.

Khan and et.al [7], proposed a novel error-free (infinite-precision) architecture for the fast implementation of both the transform 2-D Discrete Cosine Transform and Inverse DCT.

\section{III.METHODOLOGY}

\section{A. Discrete Cosine Transform (DCT):}

The DCT represents an image as a sum of sinusoids of varying magnitudes and frequencies. DCT has the property that, for a typical image most of the visually significant information about an image is concentrated in just few coefficients of DCT.The image is divided into 8x8 pixel blocks and 2D- DCT is applied to each. The DCT is a lossy compression scheme in which an NxN image block is transformed from the spatial domain to the DCT domain. DCT decomposes the signal into spatial frequencies components called DCT coefficients. The lower frequency DCT coefficients appear toward the upper left-hand corner of the DCT matrix and the higher frequency coefficients are in the lower right-hand corner of the DCT matrix. The DCT represents the frequency domain as a combination of cosine functions in the $\mathrm{x}$ and $\mathrm{y}$ directions. The two-dimensional DCT for a square $\mathrm{N} x \mathrm{~N}$ matrix is defined in equation (1)

$$
C(u, v) C(u, v)=\alpha(u) \alpha\left(\alpha(u) \alpha(v) \sum_{x=0}^{N-1} \sum_{y=0}^{N-1} f(x, y) \cos \left[\frac{\pi(2 x+1) u}{2 N}\right] \cdot \cos \left[\frac{\pi(2 y+1) v}{2 N}\right]\right.
$$

Where,

$C(u, v)$ is the coefficient of the DCT matrix at Point $(u, v)$,

$f(x, y)$ is the spatial domain value at a coordinate $(x, y)$ of the JPEG image array, $\mathrm{N}$ is the width and height of the image, and $\alpha(u), \alpha(v)$ is normalization constants.

\section{B. Arai, Agui, and Nakajama (AAN) DCT ALGORITHM:}

After the DCT is calculated, the data can be reduced to concentrate the important information into a few DCT results, leaving the remaining coefficients equal to zero. That means that the image energy is concentrated in a few DCT coefficients. To minimize the amount of calculations, the Arai, Agui, and Nakajama 8-point DCT Algorithm is used. Signal flow diagram of arai algorithm as shown in Fig.1 


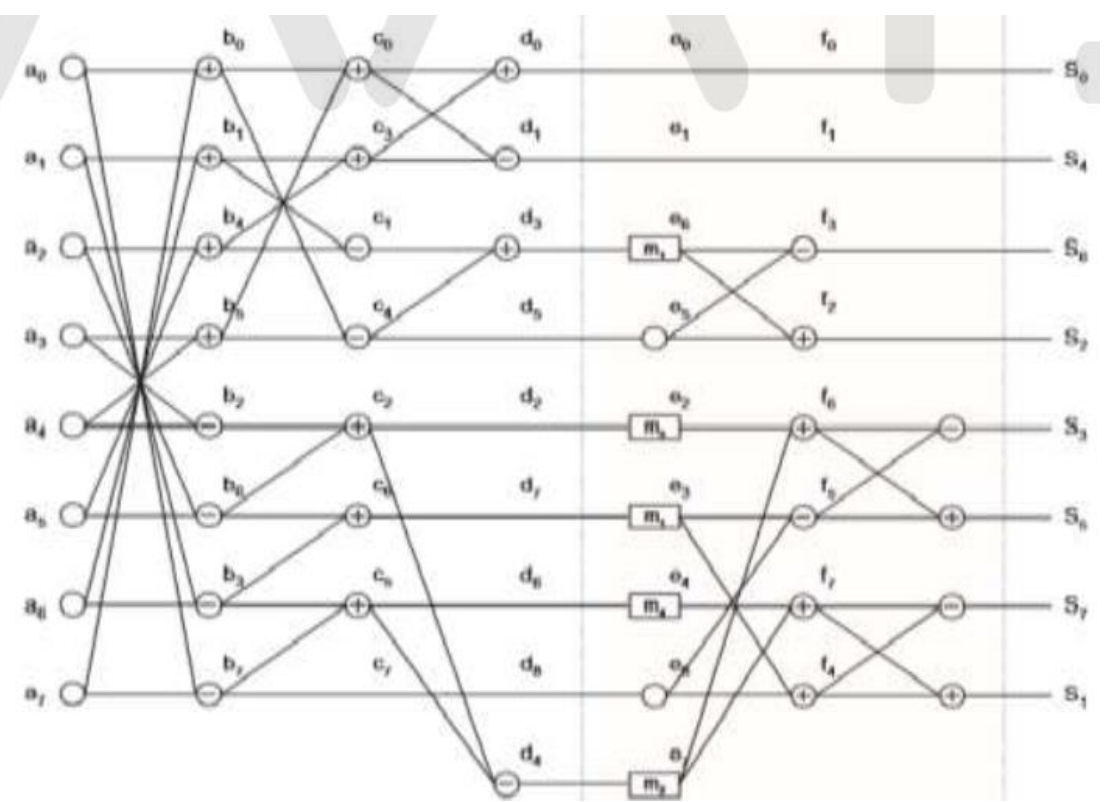

Fig.1 signal flow diagram of arai algorithm

Step 1:

$b(0):=a(0)+a(7)$

$b(1):=a(1)+a(6) ; b(2):=a(3)-a(4)$

$b(3):=a(1)-a(6) ; b(4):=a(2)+a(5) ; b(5):=a(3)+a(4)$;

$b(6):=a(2)-a(5) ; b(7):=a(0)-a(7)$

Step 2:

$c(0):=b(0)+b(5) ; c(1):=b(1)-b(4)$

$c(2):=b(2)+b(6)$

$c(3):=b(1)+b(4) ; \quad c(4):=b(0)-b(5) ; c(5):=b(3)+b(7) ;$

$c(6):=b(3)+b(6) ; c(7):=b(7)$

Step 3:

$d(0):=c(0)+c(3) ; d(1):=c(0)-c(3)$

$\mathrm{d}(2):=c(2) ; d(3):=c(1)+c(4) ;$

$d(4):=c(2)-c(5)$;

$d(5):=c(4) ; d(6):=c(5) ; d(7):=c(6)$

$d(8):=c(7)$

Step 4:

$e(0):=d(0) ; e(1):=d(1)$

$e(2):=m(3) * m(2) ; e(3):=m(1) * m(7)$;

$e(4):=m(4) * m(6) ; e(5):=d(5)$; 
$e(6):=m(1) * m(3)$

$e(7):=m(2) * m(4) ; e(8):=d(8)$

Step 5:

$$
\begin{aligned}
& f(0):=e(0) ; f(1):=e(1) ; f(2):=e(5)+e(6) ; \\
& f(3):=e(5)-e(6) ; f(4):=e(3)+e(8) ; f(5):=e(8)-e(3) ; \\
& f(6):=e(2)+e(7) ; f(7) s:=e(4)+e(7)
\end{aligned}
$$

Step 6:

$s(0):=f(0) ; s(1):=f(4)+f(7) ;$

$s(2):=f(2) ; s(3):=f(5)-f(6)$;

$s(4):=f(1) ; \quad s(5):=f(5)+f(6)$;

$s(6):=f(3) ; s(7):=f(4)-f(7)$;

Where

a(i),y(i) are input and output data;

$\{\mathrm{Si}\}$ are scaled DCT coefficient

For $\mathrm{i}$ in 0 to 7 loop

$y(i):=s a(i) * s(i) * 4.0$;

End loop;

$$
\begin{aligned}
& m 1=\cos \left(\frac{\pi}{4.0}\right) ; m 2=\cos \left(\frac{\pi * 3}{8}\right) ; \\
& m 3=\cos \left(\frac{\pi}{8}\right)-\cos \left(\frac{\pi * 3}{8}\right) ; m 4=\cos \left(\frac{\pi}{8}\right)+\cos \left(\frac{\pi * 3}{8}\right) ; \\
& s(0)=\frac{0.5}{\operatorname{sqrt}(2)} ; s(i)=\frac{0.25}{\cos \left(\pi * \frac{i}{16}\right)}, i=1,2, \ldots . .7 .
\end{aligned}
$$

\section{Quantization:}

Data from DCT output is quantized by a quantizer. Quantization is done by dividing each DCT coefficient by corresponding quantizer step size followed by rounding to nearest integer by using equation (2).

$$
F^{Q}(u, v)=\text { Integer Round }\left(\frac{F(u, v)}{Q(u, v)}\right)
$$

DCT-based image compression relies on two techniques to reduce the data required to represent the image. The first is quantization of the image's DCT coefficients; the second is entropy coding of the quantized coefficients. Quantization is the process of reducing the number of possible values of a quantity, thereby reducing the number of bits needed to represent it. Entropy coding is a technique for representing the quantized data as compactly as possible.

\section{Huffman Coding:}

Proposed by DR. David A. Huffman in 1952. - A method for the construction of minimum redundancy code. Huffman code is a technique for compressing data. Huffman's greedy algorithm looks at the occurrence of each character and it as a binary string in an optimal way. Huffman coding is a form of statistical coding which attempts to reduce the amount of bits required to represent a string of symbols. The algorithm accomplishes its goals by allowing symbols to vary in length. Shorter codes are assigned to the most frequently used symbols, and longer codes to the symbols which appear less frequently in the string (that's where the statistical part comes in). Code word lengths are no longer fixed like ASCII. Code word lengths vary and will be shorter for the more frequently used characters. 


\section{IV.PROPOSED APPROACH}

The image compression procedure using DCT is as shown in Fig. 2 and its steps are as follows,

1. The image is divided into $8 \times 8$ blocks of pixels.

2. It works from left most to right most corners; top to bottom, the DCT can be applied to each block of image.

3. The algebraic integer based on the Arai, Agui, and Nakajima (AAN) algorithm is used to reduce the computational complexity.

4. Then, each block is compressed by performing quantization process.

5. The collection of the compressed blocks that represent the image is stored in a hugely reduced amount of space.

6. Finally, the quantized DCT coefficients are sent to the entropy encoder such as Huffman encoder. The output is a sequence of compress data.

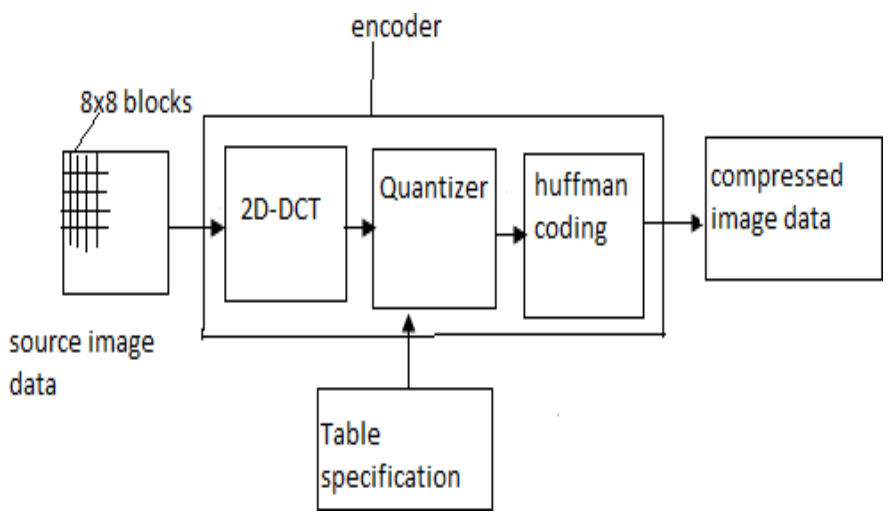

Fig.2 compression procedure

SYNTHESIS AND SIMULATION RESULT

The synthesis and simulation is done in Xilinx ISE 9.1i.

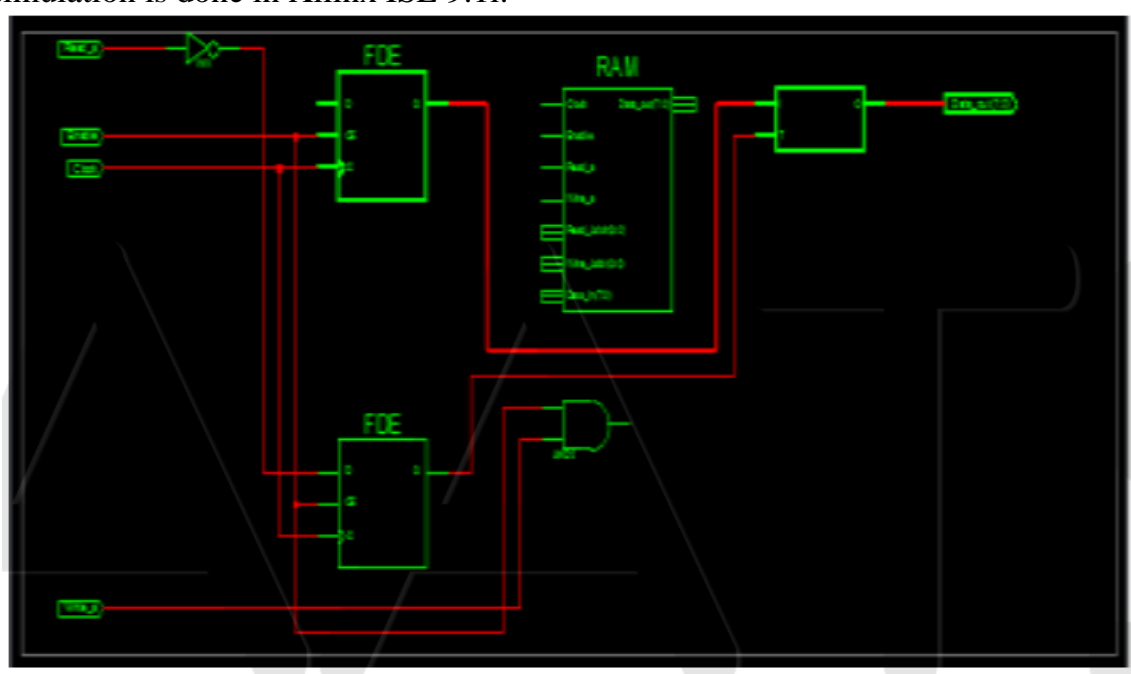

Fig.3 RTL view of RAM using TEXT I/O

The RTL view of RAM using TEXT I/O, which consists of RAM, used for storing the input output text is as shown in Fig.3

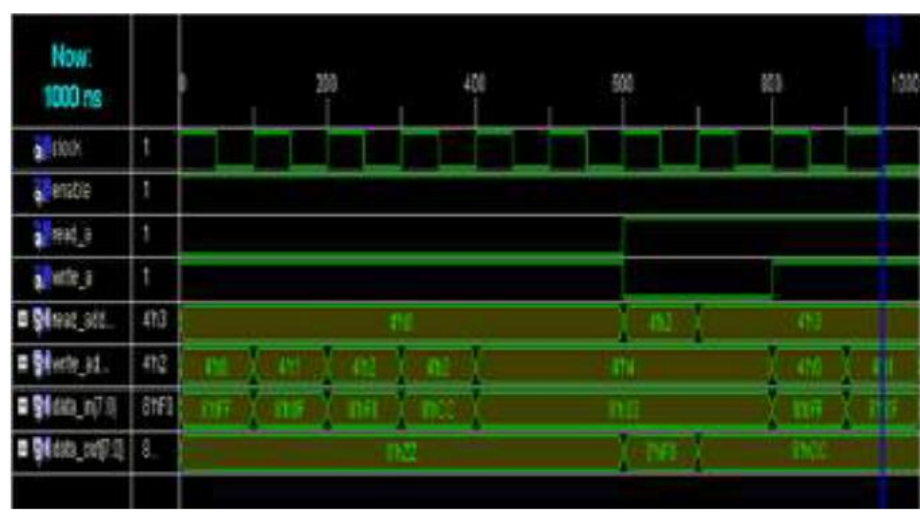

Fig.4 Simulation result of RAM using Text IO 
Fig.4 shows the Simulation result of RAM using Text IO. The text I/O is used for write address and data in text and read data from their respective addresses.

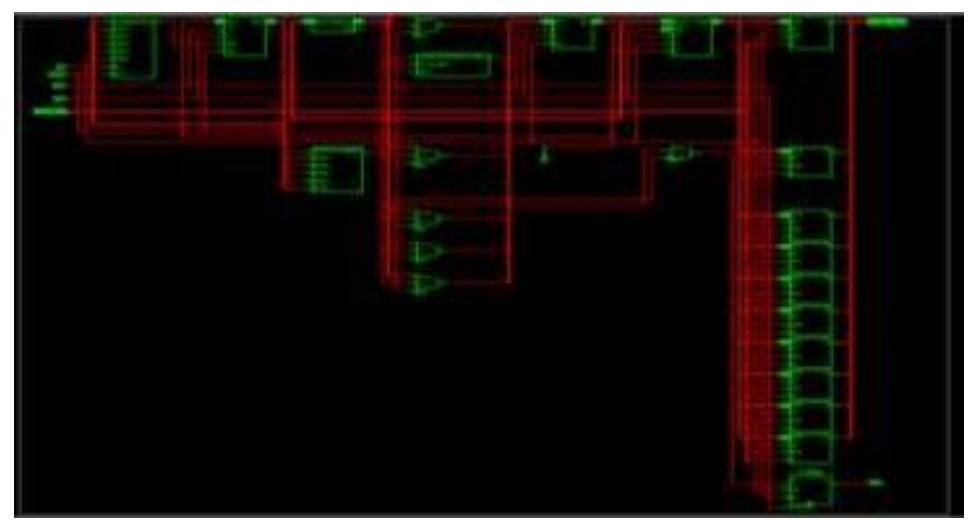

Fig. 5 RTL view of DCT Buffer

The DCT buffer is used to store the data in first in first out (FIFO) manner. The RTL view of DCT Buffer is as shown in Fig. 5

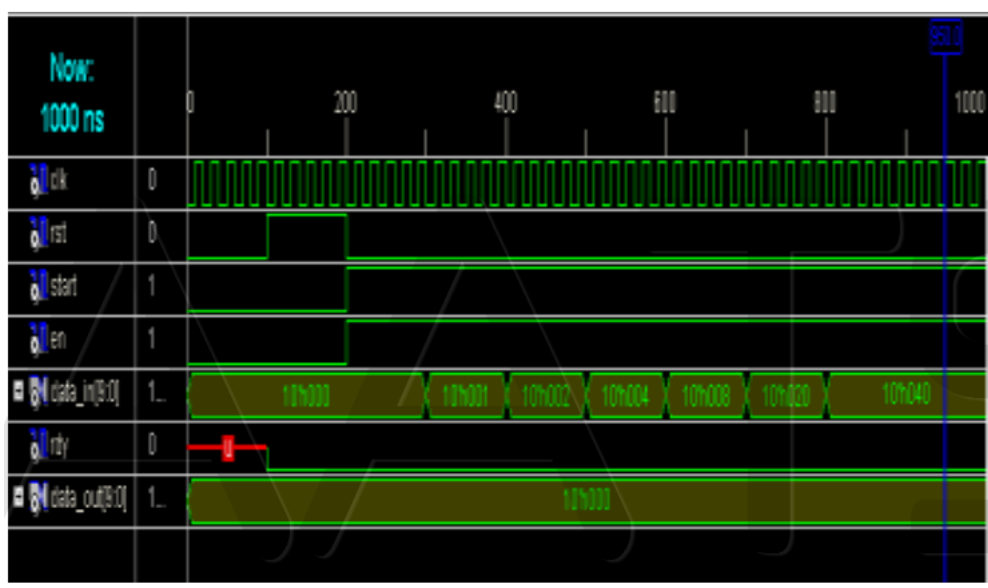

Fig.6: Simulation of DCT buffer

Fig.6 shows the simulation of DCT buffer.The function of this buffer, 64 (arri) data are stored to the FIFO buffer and this data read from FIFO taps in the transposed order.

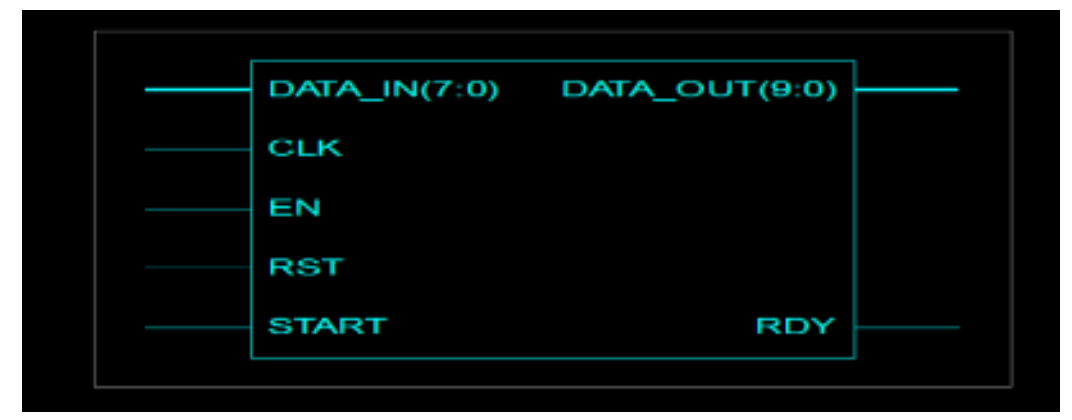

Fig.7 RTL view of Arai, Agui, and Nakajima (AAN) algorithm

Fig.7 shows the RTL view of Arai, Agui, and Nakajima (AAN) algorithm,which consists of five input ports DATA_IN of 8bits,CLK, EN, RST and START and two outputs DATA_OUT of 10 bits and RDY. 


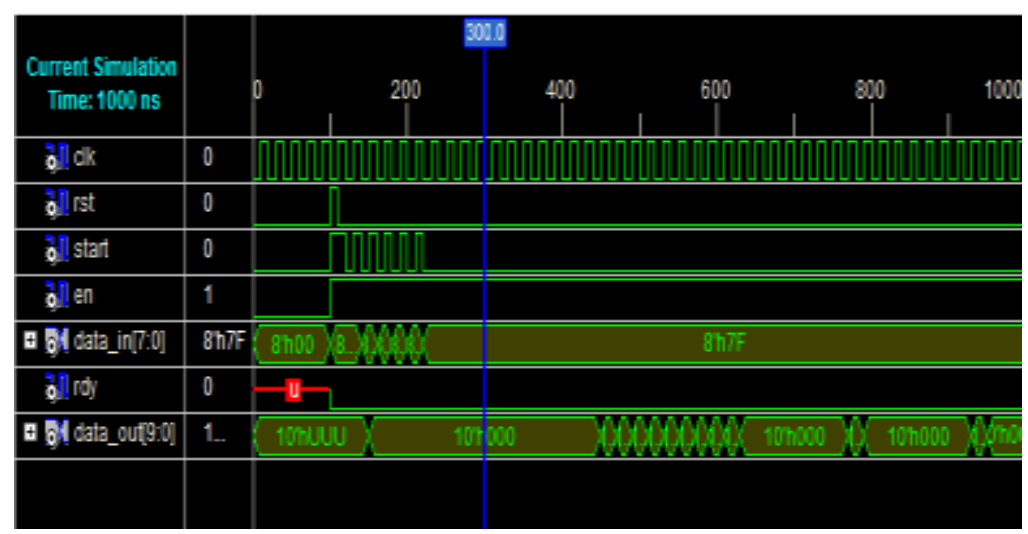

Fig.8 Simulation of Arai, Agui, and Nakajima (AAN) algorithm

Fig. 8 shows the simulation of Arai, Agui, and Nakajima (AAN) algorithm, which calculate the multiplication factor and scaled DCT coefficients.

\section{CONCLUSION}

In this paper, RAM using Text I/O, DCT Buffer and Arai, Agui, and Nakajima (AAN) algorithm are designed in VHDL. Here, we have introduced an encoding scheme for the compression of the image which provides the low complexity architecture based on 8-point Arai algorithm. For reducing the size of the data sent i.e. image compression, to compute 2-D DCT with scaled output mode which effectively reduces the overall arithmetic and multiplication operations. This method is fast and provided the low complexity.

\section{REFERENCES}

[1]. Fausto Galvan, Giovanni Puglisi, Arcangelo Ranieri Bruna, and Sebastiano Battiato,“ First Quantization Matrix Estimation From Double Compressed JPEG Images," IEEE Transactions On Information Forensics And Security, Vol. 9, No. 8,pp, 1299-1310, August 2014.

[2]. Hamdy M. Mousa, Mostafa A. Ahmad, and Ashraf B. El-Sisi,“ Image Compression Ratio Enhancement Based on Conformal Mapping," 978- 1-4799-0080-0/13,pp 280-285,IEEE 2013.

[3]. Arjuna Madanayake, Renato J. Cintra, , Denis Onen, Vassil S. Dimitrov, Nilanka Rajapaksha, L. T. Bruton, and Amila Edirisuriya, "A Row- Parallel 8×8 2-D DCT Architecture Using Algebraic Integer-Based Exact Computation”, IEEE Transactions On Circuits And Systems For Video Technology, Vol. 22, No. 6, PP. 915-929,June 2012.

[4]. Smitha Joyce Pinto and Prof. Jayanand P.Gawande, "Performance analysis of medical image compression techniques", 978-1-4673-2590-5/12, IEEE 2012.

[5]. H. L. P. Arjuna Madanayake, R. J. Cintra, D. Onen, V. S. Dimitrov and L. T. Bruton, “Algebraic Integer based $8 \times 8$ 2-D DCT Architecture for Digital Video Processing”, 978-1-4244-9474-3/11,PP. 1247-1250,IEEE 2011.

[6. Hatim Anas, Said Belkouch, M. El Aakif and Noureddine Chabini, "FPGA Implementation Of A Pipelined 2D-DCT And Simplified Quantization For Real-Time Applications", 978-1-61284-732-0/11/\$, IEEE 2010.

[7]. K. Wahid, V. Dimitrov, and G. Jullien, "Error-free computation of $8 \times 8$ 2D DCT and IDCT using two-dimensional algebraic integer quantization," in Proceedings of the 17th IEEE Symposium on Computer Arithmetic. IEEE Computer Society, Jun. 2005, pp. 214-221.

[8]. Arai, Y., Agui, T., and Nakajima, M.: ‘A fast DCT-SQ scheme for images', Trans. IEICE, 1988, E71, (11), pp. 10951097

[9]. Rafael C. Gonzalez and Richard E. Woods, Digital Image Processing,3rd edn, Prentice Hall publication, South Asia,2009.

[10]. Jayaram. Bhasker, A VHDL Primer, $3^{\text {rd }}$ edn, Prentice Hall publication, 2003. 